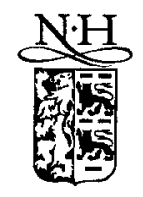

ELSEVIER

\title{
Electromagnetic fields in two-dimensional models of near-field optical microscope tips
}

\author{
Annick Castiaux ${ }^{a}$, Alain Dereux ${ }^{\text {a }}$, Jean-Pol Vigneron ${ }^{\text {a }}$, Christian Girard ${ }^{b}$, \\ Olivier J.F. Martin ${ }^{c}$ \\ a Institute for Studies in Interface Sciences, Facultés Universitaires Notre-Dame de la Paix, Rue de Bruxelles 61, B-5000 Namur, Belgium \\ b Laboratoire de Physique Moléculaire UA CNRS 772, Université de Franche Comté, F-25030 Besançon Cedex, France \\ c Institute for Field Theory and High Frequency Electronics, Swiss Federal Institute of Technology, ETH-Zentrum, \\ CH-8092 Zurich, Switzerland
}

Received October 1993*; in final form 31 August 1995

*Originally submitted for the proceedings of NFO 2 (Ultramicroscopy 57, Nos. 2/3)

\begin{abstract}
The design of tips is of outstanding importance to obtain maximal efficiency in scanning near-field microscopy. As a support towards this optimization, this work presents the computation of the electromagnetic field penetrating into twodimensional tips. The investigated geometries consist of elongated two-dimensional dielectric slabs (glass) terminated by pointed tips. Distributions of the electric field amplitude inside and outside two-dimensional models of tips are computed and from those solutions, far-field differential cross-sections are obtained.
\end{abstract}

\section{Introduction}

In the early eighties, the exploitation of near-field effects expanded the resolution of optical microscopy down to the mesoscopic dimensions $(10-100 \mathrm{~nm})$ [1]. Since then, a broad variety of scanning near-field optical microscopes (SNOM) have been elaborated. Different designs were tested with the aim of improving the SNOM performances. Apertures [2] and pointed optical fibres $[3,4]$ were successfully developed. Sample images were recorded under reflection, transmission and tunneling illuminations $[2,4,5]$. Resonance techniques based on localized plasmons [6] or molecular fluorescence excitations [7] were introduced. Recently, opaque probe tips [8] were used to reach a resolution $(\sim 3 \mathrm{~nm})$ close to the electron scanning tunneling microscope.

During this initial era of rapid technological devel- opments, little attention was paid to a deep understanding of electromagnetic near-field phenomena in order to contribute to instrumental improvements. Particularly, the study of tip shapes and compositions remained limited to phenomenological considerations. Although most experiments detect the far-field issued from near-field effects, the relation between far-field intensities and the electromagnetic field distribution inside, as well as around the tip, was not systematically investigated. Preliminary studies of this problem were undertaken only very recently [9]. This is not only due to the fact that direct measurement of the near-field is impossible without disturbing the investigated electromagnetic field distribution but also to the difficult mathematical challenge raised by solving Maxwell's equations in the awkward geometries typical of SNOM devices.

Recently a method to solve the vectorial wave 
equation in such geometries was finalized by Martin, Dereux and Girard [10]. The method involves Green-functions and is based upon the simultaneous iteration of Lippman-Schwinger's and Dyson's equations [11]. This approach allows one to describe the propagation of the electromagnetic field when dealing with localized perturbing objects (dielectric or metallic). The method has a precious advantage: no symmetry constraints are required. Perturbations of arbitrary shape and composition are tractable. The accuracy of the method depends only on the density of the grid used to discretize the perturbations. This versatility is essential in the study of SNOM tips facing corrugated surfaces.

In our work, this method was implemented for twodimensional problems. The only approximation introduced in these computations was the discretization of the perturbations in small meshes. So it was really important to be accurate when choosing the discretization step. Realistic computations are obtained when the step is at least ten times smaller than the effective wavelength $\lambda_{\text {inc }} / n$, where $n$ is the real part of the refraction index of the perturbation. Thus, the number of perturbation meshes can be very high. However the procedure used for these $2 \mathrm{D}$ scalar computations is rather rapid. In this particular work, the longest computation took about an hour. All computations were performed on RS-6000 IBM workstations.

Step by step we show the transition from a simple glass slab to a tapered and coated tip probe as those used in near-field microscopy to illuminate a sample and studied the coupling of this kind of tip with a surface. The basic considered structure is a truncated slab waveguide. Then we sharpen the extremity of the slab to obtain a first model of SNOM tip and we cover the boundary faces of the slab with a metal coating. From the near-field results we compute the far-field crosssections relevant for comparison with experimental results. Finally we study the interaction between a tip and a small defect on a surface. An electric field polarized parallel to the interfaces and dielectric functions taken from the literature $[12,13]$ fix our working conditions. We use bulk dielectric functions which are known to be good approximations for very thin layers $[14,15]$.

The near-field distributions discussed in this work are presented as contour plots of the electric field amplitude. If $A_{\mathrm{m}}$ is the maximal amplitude, the plain con- tours specify amplitudes larger than $A_{\mathrm{m}} / 2$, the dashed contour designates an amplitude equal to $A_{\mathrm{m}} / 2$ and the dotted contours mean amplitudes smaller than $A_{\mathrm{m}} / 2$. Each contour line represents a defined amplitude. In the angular plots of far-field cross-sections, zero angle is the direction of forward scattering.

\section{Diffraction by isolated 2D tips}

In the results presented in this section, plane waves are incident from the left on the structures under study. The structures are glass slabs having a thickness of $0.55 \mu \mathrm{m}$. When a metal coating is added, it is a gold coating $0.05 \mu \mathrm{m}$ thick.

\subsection{Truncated slab waveguide (Figs. 1 and 2)}

The investigated configuration is a rectangular glass core embedded in air. The slab is $3.0 \mu \mathrm{m}$ long. This choice was dictated by two reasons. First the slab is long enough to support longitudinal eigenmodes. Second it is short enough to comply with the requirement of localized perturbation associated with our Green's function approach.

For an incident wavelength $\left(\lambda_{\text {inc }}\right)$ of $1 \mu \mathrm{m}$ close to the laser transition in a neodymium glass laser, the incident wavelength is twice the slab thickness so that, at first sight, the slab behaves as a monomode waveguide (Fig. 1a). However analytical results show that two modes are supported by an infinite slab with the same thickness and refraction index. In our computation, displaying the amplitude prevents us from distinguishing them. Since the slab is of finite length, the larger amplitudes (plain contours) concentrated inside the slab correspond to stationary waves. Just outside the truncated slab the electric field is decreasing drastically. The near-field output amplitude at the exit termination of the slab is significantly large.

Keeping the original dimensions of the dielectric object, we tune the wavelength to $488 \mathrm{~nm}$ (Argon laser). This wavelength is somewhat smaller than the slab thickness. Consequently, the distribution of the near-field amplitudes (Fig. 1b) is more complicated than in the previous case since more modes are now allowed to propagate inside the slab. However the wave is still well guided within the slab. The spatial width of the output amplitude when exiting the truncated slab is narrower than for $\lambda_{\text {inc }}=1 \mu \mathrm{m}$. 

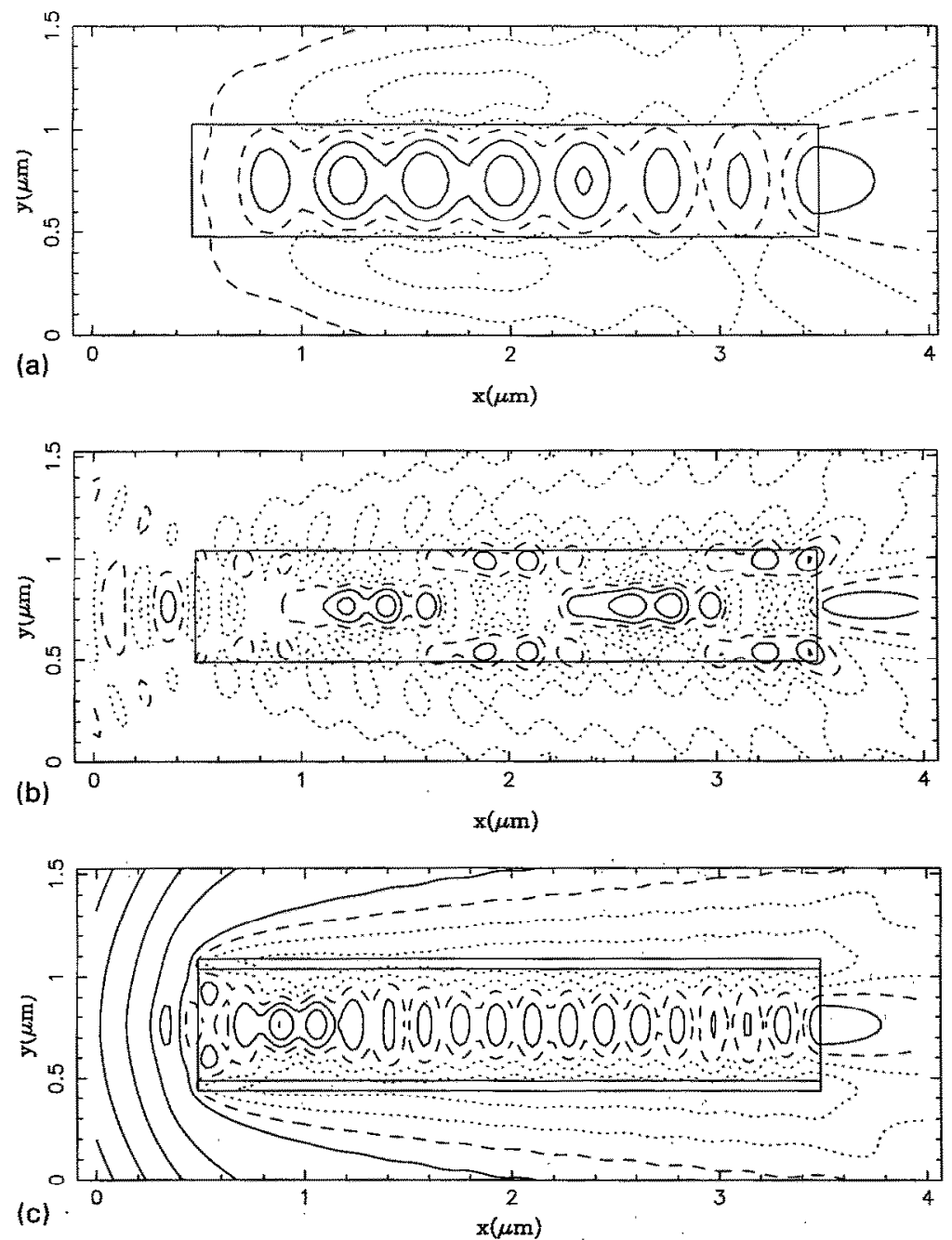

Fig. 1. Truncated slab waveguide $(3 \mu \mathrm{m} \times 0.55 \mu \mathrm{m})$, computed near-field distributions. A plane wave incoming from the left side is propagating along the $z$-axis. (a) $\lambda_{\text {inc }}=1 \mu \mathrm{m}$ and $\varepsilon_{\text {slab }}=2.10 ;(b, c) \lambda_{\text {inc }}=488 \mathrm{~nm}$ and $\varepsilon_{\text {slab }}=2.14 ;$ (c) A gold coating is added on both horizontal sides $\left(\varepsilon_{\text {gold }}=(-2.2,4.02)\right)$.

The introduction of boundary conditions corresponding to a vanishing field at the horizontal surfaces limiting the slab can force the fundamental eigenmode even for the green incident wavelength. A possible implementation is shown in Fig. 1c. As a result of the deposition of a metallic coating on the horizontal surfaces, the near-field structure is deeply modified. A regular field structure is established and a more important reflection of the incident wave is observed. The presence of a metallic coating does not alter significantly the spatial width of output amplitude at the exit of the slab.

The far-field cross-sections issued from the nearfield distributions are important since they are related to experimentally accessible data (Fig. 2). It turns out that the above mentioned collimation of the output wave also appears in the Fraunhofer zone. This is related to the well known Mie effect for small particles. The forward scattering is much more intense for $\lambda_{\text {inc }}=488 \mathrm{~nm}$ (dashed curve) than for $\lambda_{\text {inc }}=1$ $\mu \mathrm{m}$ (plain curve). This is conceivably due to the easier penetration of this shorter wavelength into the slab 


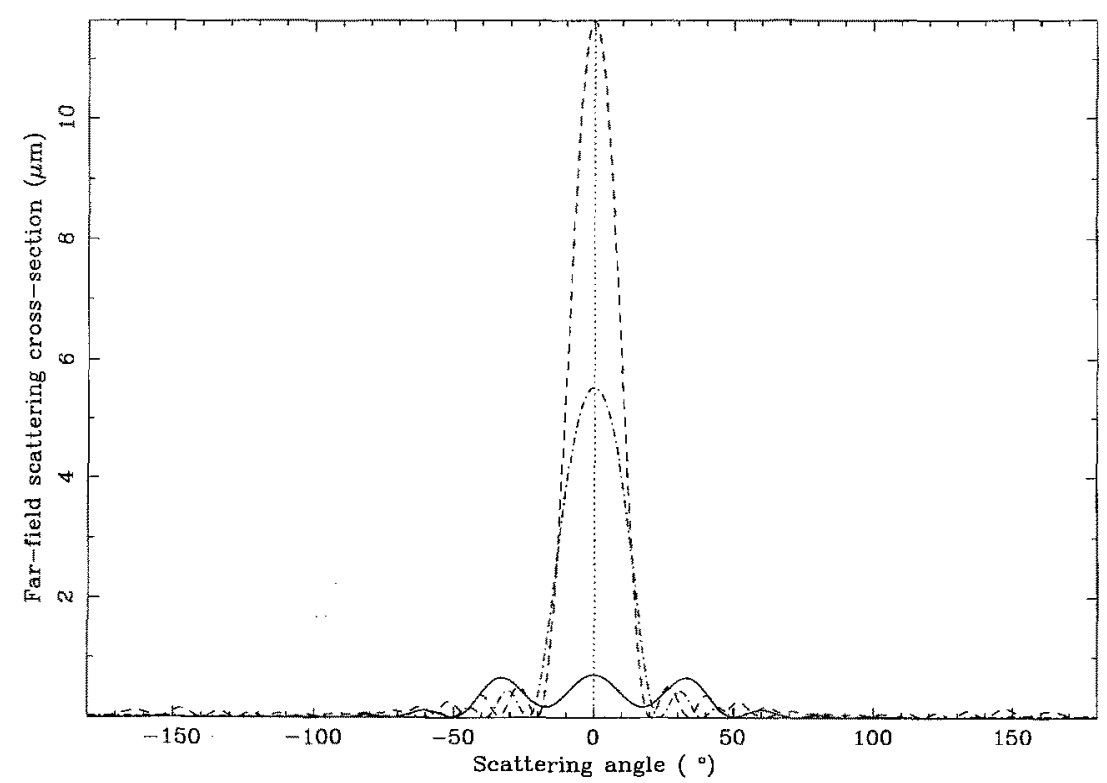

Fig. 2. Truncated slab waveguide ( $3 \mu \mathrm{m} \times 0.5 \mu \mathrm{m}$ ). Computed far-field differential cross-sections. The plain curve corresponds to Fig. la, the dashed one corresponds to Fig. $1 \mathrm{~b}$ and the dashed-dotted one corresponds to Fig. 1c.

which enhances the Mie effect. The coupling of the incident wavelength to a finite size dielectric structure is not a trivial problem and has been underestimated in the development of SNOM tips. Indeed, for a given geometry, we realized that this coupling can lead to totally different characteristics according to the wavelength. For instance, we observed that coating the slab enhances the forward peak at $\lambda_{\text {inc }}=1 \mu \mathrm{m}$. On the contrary, the output intensity decreases at $\lambda_{\text {inc }}=488$ $\mathrm{nm}$ (dashed-dotted curve). The presence of the coating fixes new boundary conditions which, according to the wavelength, are favourable or not to the coupling between the incident plane wave and the dielectric slab. On the other hand the slight broadening of the forward peak occurs for any wavelength. This is a diffraction effect. The width of the peak is comparable to that of the central peak when a wave is diffracted by a slit having the same width as the slab.

\subsection{Sharpening the extremity of the slab}

Following the intuitive procedure used by experimentalists, we make a tip by sharpening the extremity of the precedent slab structure. The aim of such a sharpening is to increase the output collimation so that the zone scanned by such a SNOM tip is reduced laterally, leading to an improved resolution. The same two incident wavelengths $(1 \mu \mathrm{m}$ and $488 \mathrm{~nm}$ ) are considered. As we already observed in the slab geometry, the near-field distribution inside the tip is more complicated for the small incident wavelength (Fig. 3b) then for the larger one (Fig. 3a). When the wavelength is $1 \mu \mathrm{m}$, a single collimated spot comes out the tip. However, when tuning the wavelength to $488 \mathrm{~nm}$, not only the end corner but also the lateral corners of the tip are responsible for near-field high amplitudes. Such multiple beams were already observed experimentally and present a problem for the interpretation of images. To eliminate these lateral lobes which can lead to ambiguous interpretation of SNOM images, a coating is deposited on the boundary surfaces of the slab, including the lateral corners of the tip. We obtain an aperture of $150 \mathrm{~nm}$ at the apex of the tip. The resulting near-field distribution is given in Fig. $3 c$, where the lateral lobes have been forced out.

The far-field cross-sections (Fig. 4) associated to the near-field patterns of Fig. $3 \mathrm{~b}$ and Fig. $3 \mathrm{c}$ show also these lateral lobes when the tip is not coated (plain curve) and the decay of the lobes when a coating is added (dashed curve). In both cases the envelope is modulated by diffraction maxima and minima. We can also compare these curves to the ones for the truncated 

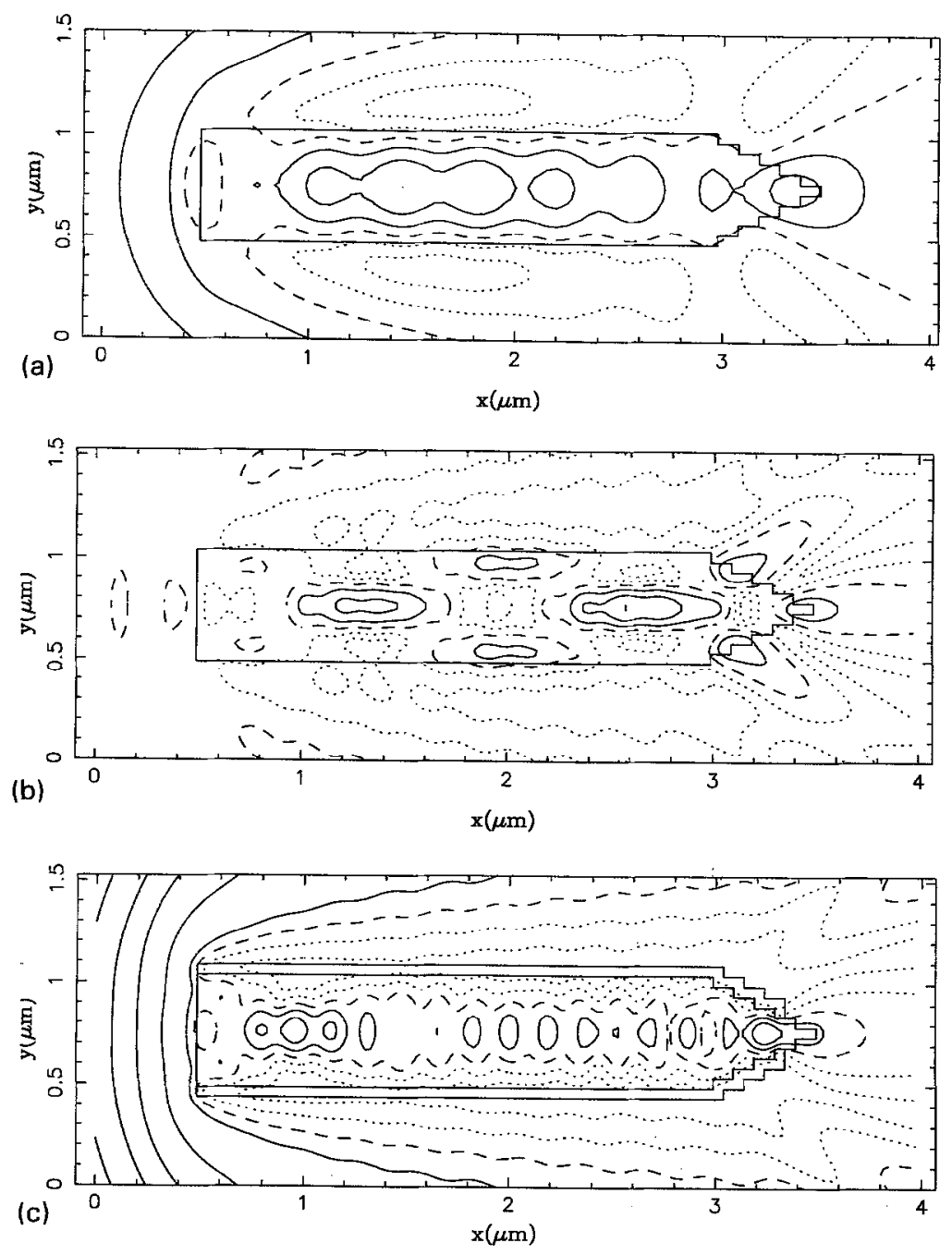

Fig. 3. Sharpened slab, computed near-field distributions. Same conditions as Figs. 1a, 1b and Ic, respectively.

slab geometry (Fig. 2, dashed curve). The central peak is slightly collimated when the slab is sharpened but its power loss is transferred to the lateral lobes. The sharpening helps the collimation but reduces the power of the outgoing beam. It is necessary to obtain a compromise between the output power and the collimation.

\section{Application: tip-defect interaction}

Such two-dimensional simulations were already accomplished by other teams using different methods. Novotny [9] studied how the near-field distributions are modified when a small defect is moved on a surface in front of which he placed a tip. Here we want to consider how the presence of a slight geometrical defect on a surface can modify the near-field as well as the far-field, following the lateral position of this defect. The considered configuration is an uncoated illuminating two-dimensional tip in front of a surface. All elements are in glass, in such a way that only geometrical effects are taken into account. To enhance the collimation, the incident wave is a gaussian beam having a FWHM of $1.5 \mu \mathrm{m}$ and an incident wavelength of $633 \mathrm{~nm}$. To isolate the tip-sample interaction, we put a silver screen at the bottom of the slab. 


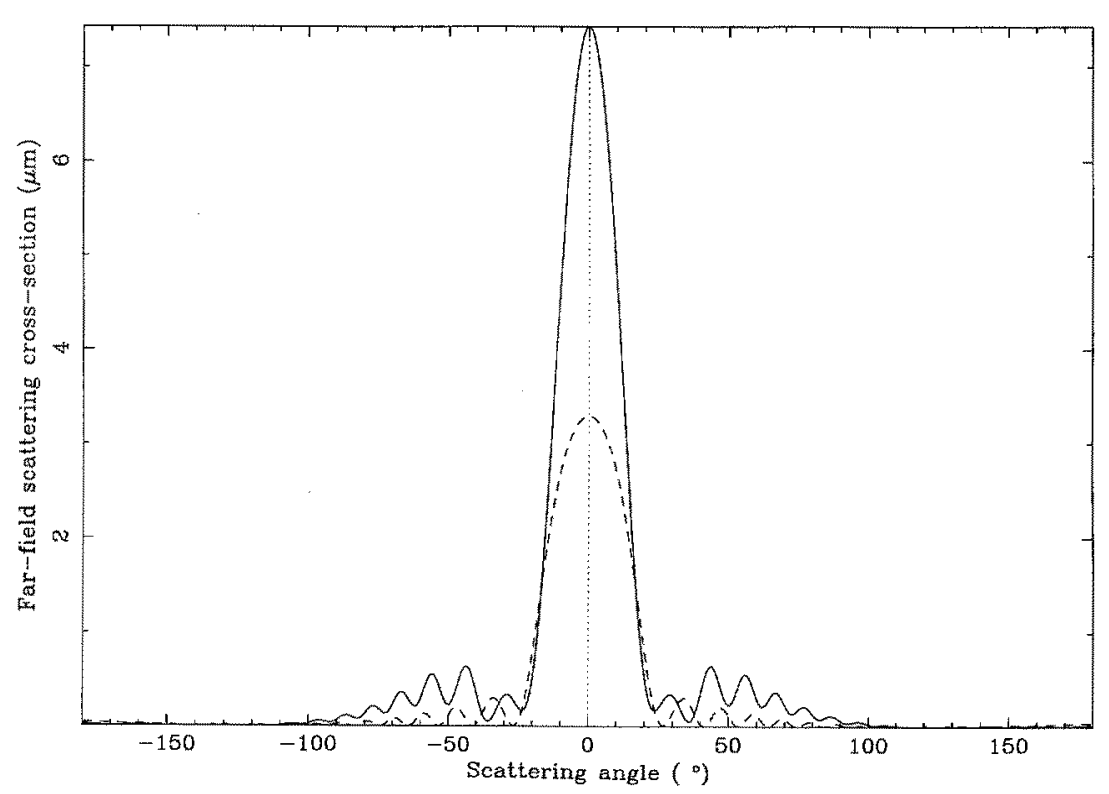

Fig. 4. Effect of a coating for a sharpened slab, computed far-field differential cross-sections. The continuous curve corresponds to Fig. $3 \mathrm{~b}$ and the dashed one corresponds to Fig. $3 \mathrm{c}$.

Fig. 5 shows near-field patterns computed when there is (Fig. 5b) or not (Fig. 5a) a tiny protuberance $150 \mathrm{~nm}$ on the surface. The protuberance dimensions are as follows: it is $150 \mathrm{~nm}$ broad and $100 \mathrm{~nm}$ high. The protuberance is laterally $100 \mathrm{~nm}$ far from the apex of the tip. The perfect symmetry of the near-field distribution in Fig. $5 a$ is broken by the introduction of the defect. The steady-state waves in the fibre are perturbed and an optical bridge is created between the apex of the tip and the defect. The change induced by the presence of a defect on the surface is enhanced in Fig. $5 c$ where the amplitude of the difference between the electric field with and without the defect was computed. The larger difference is concentrated in the defect and propagates beyond the metallic screen as well as through the slab. The same kind of representation is also given in Fig. 6 when the defect is right in front of the tip apex. Here, of course, the symmetry is conserved, but the defect induced differences in the near-field pattern. These differences are more intense and their influence in reflection and transmission is more sensible than when the defect is laterally shifted. The presence of the defect could be detected under reflection or transmission but the transmission mode is more favourable in this configuration because in the reflection mode the presence of the tip and the screen modifies the reflected wave.

The curves presented in Fig. 7 are describing the far-field cross-section for the three cases discussed above. In comparison with the far-field when there is no defect on the surface (plain curves), one observes that the presence of the defect causes a slight loss in reflection and a slight gain in transmission. The position of the defect is not easy to discriminate. One can see that the dotted-dashed curve - corresponding to the asymmetric instance - is slightly asymmetric, if we consider the lateral angles. It seems that the detection would be easier in transmission. However, no definitive conclusion can be drawn out these computations about the best detection angle for two reasons. First, the back scattering is enhanced by the presence of the metal screen at the bottom of the tip. Secondly, the 2D structure is finite in the $x$-direction and border effects interfere in the far-field representation.

\section{Conclusions}

In this paper, we presented two-dimensional computations of scattering phenomena occurring near and inside near-field probes used as emitters. In three steps, we described the transition from a simple glass slab 


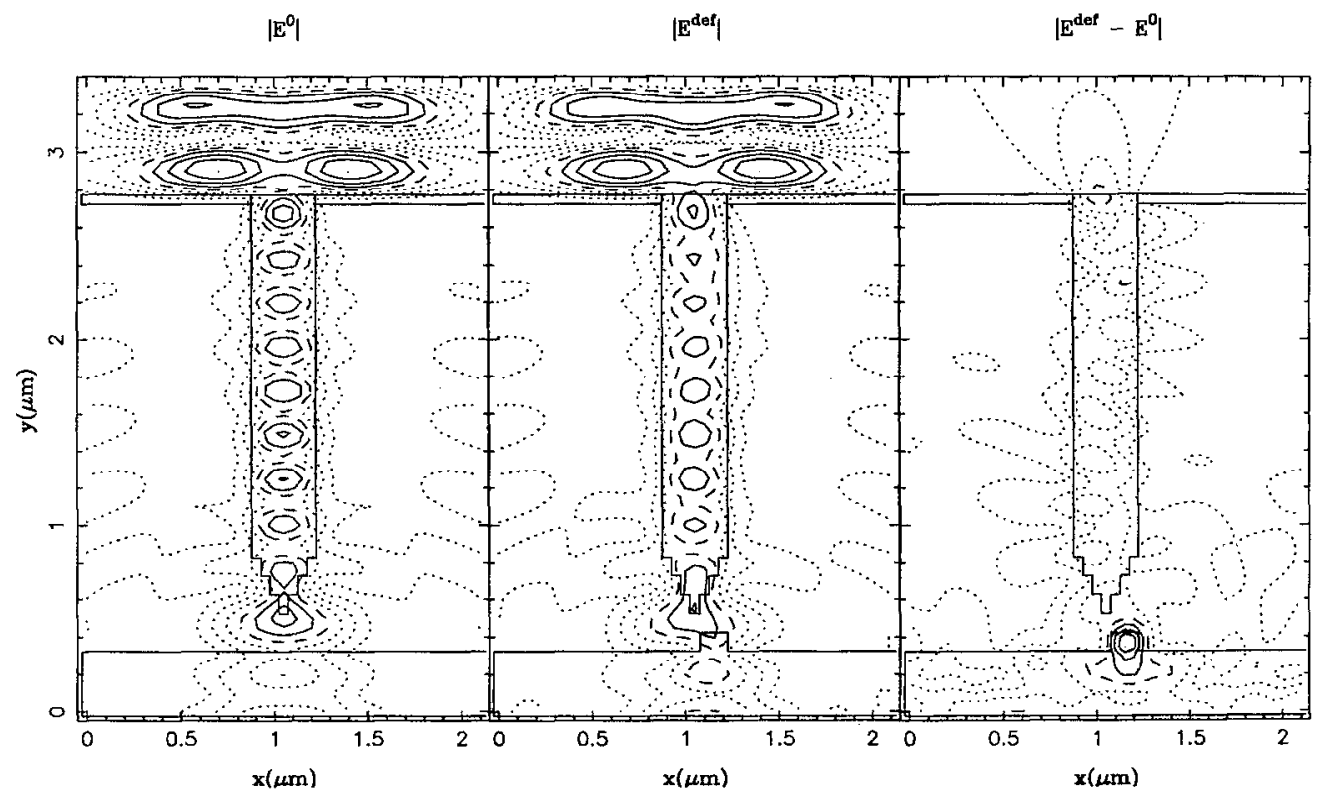

Fig. 5. Coupling of a 2D tip with a finite sample, effect of a laterally shifted defect. A collimated wave is incident from the top (FWHM $=$ $1.5 \mu \mathrm{m}$ and $\left.\lambda_{\text {inc }}=633 \mathrm{~nm}\right)$. The tip, the surface and the defect are made of glass $\left(\varepsilon_{\text {glass }}=2.12\right)$. The metal screen is made of silver $\left(\varepsilon_{\text {silver }}=(-18.0,0.5)\right)$.

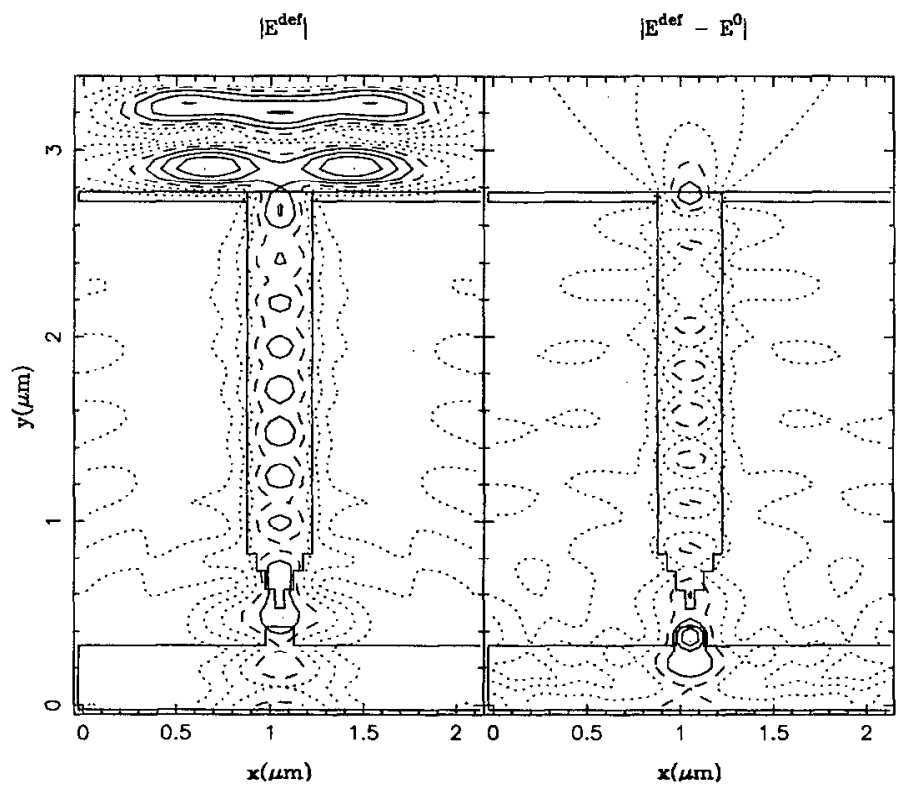

Fig. 6. Coupling of a 2D tip with a finite sample, effect of a symmetric defect. Same computations as Fig. 5 , when the defect is right in front of the tip. 


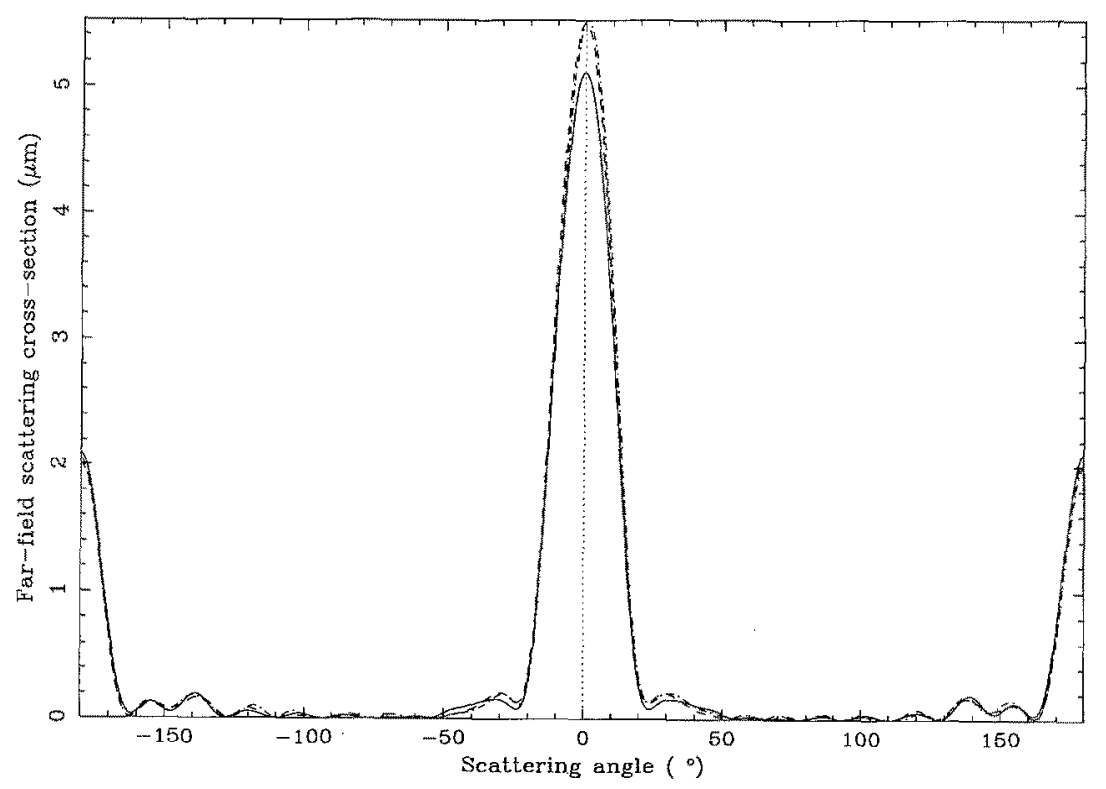

Fig. 7. Far-field cross-sections for the coupling of a 2D tip with a finite sample. The plain curve corresponds to the plane surface, the dashed curve corresponds to the surface having a geometrical defect in front of the tip, the dashed-dotted curve corresponds to the slight shift of the defect on the surface.

to a tapered and coated near-field probe, following the experimental process. We showed the utility of a metallic coating for multimodal fibres to ensure the collimation and the uniqueness of the outgoing beam when the tip is used as an emitter. We also observed that a coating does not help the collimation for a single mode fibre. However, it could be of great importance to localize the tip-surface interaction in a small zone when scanning a sample. This phenomenon is now under study. Finally, we considered the coupling occurring between such a tip and a dielectric surface with and without a small geometrical defect. We presented the modifications induced by the defect in the near-field distribution and in the far-field detection. We showed that such a defect can be easily detected in transmission or reflection.

Although three-dimensional studies along the lines followed in this paper would be more realistic, the twodimensional computations presented here already provide good representations of near-ficld bchaviours relevant to SNOM devices. Vectorial computations considering the p-polarization or three-dimensional models were also realized using the same algorithm as the one used here $[16,17]$. They consume a lot of time and computer memory. The information provided by these two-dimensional scalar models is qualitatively sufficient to understand the mechanisms taking place in near-field microscopy experiments. For quantitative results, more accurate computations using threedimensional codes are necessary.

\section{Acknowledgements}

We want to thank Daniel Courjon and Michel Spajer for very fruitful discussions about experimental results. A.C. is grateful to the National Fund for Scientific Research (F.N.R.S.) for financial support.

\section{References}

[1] D.W. Pohl, Appl. Phys. Lett. 44 (1984) 651.

[2] U.T. Dürig, D.W. Pohl and F. Rohner, J. Appl. Phys. 59 (1986) 3318 .

[3] R. C. Reddick, R. J. Warmack and T. L. Ferrell, Phys. Rev. B 39 (1989) 767.

[4] D. Courjon, J.-M. Vigoureux, M. Spajer, K. Sarayeddine and S. Leblanc, Appl. Opt. 29 (1990) 3734.

[5] E. Betzig, A. Harootunian, E. Kratschmer, A. Lewis and M. Isaacson, Bull. Am. Phys. Soc. 30 (1984) 42.

[6] U.Ch. Fischer and D.W. Pohl, Phys. Rev. Lett. 62 (1989) 458. 
[7] A. Harootunian, E. Betzig, M. Isaacson and A. Lewis, Appl. Phys. Lett. 49 (1986) 674.

[8] M. Specht, J.D. Pedarnig, W.M. Heckl and T.W. Hänsch, Phys. Rev. Lett. 68 (1992) 476.

[9] L. Novotny, D.W. Pohl and P. Regli, Ultramicroscopy 57 (1995) 180.

[10] O.J.F. Martin, A. Derenx and C. Girard, J. Opt. Soc. Am. A 11 (1994) 1075 .

[11] E.N. Economou, Green's Functions in Quantum Physics (Springer, Berlin, 1983).

[12] H. Raether, Surface Plasmons on Smooth and Rough Surfaces and on Gratings (Springer, Berlin, 1988).
[13] E.D. Palik, Handbook of Optical Constants of Solids (Academic Press, New York, 1985).

[14] Ph. Lambin, P. Senet, A. Castiaux and L. Philippe, J. Phys. I France 3 (1993) 1417.

[15] C.F. Bohren and D.R. Huffman, Absorption and Scattering of Light by Small Particles (Wiley, New York, 1983) ch. 12.

[16] O.I.F. Martin, C. Girard and A. Dereux, Phys. Rev. Lett. 74 (1995) 526.

[17] C. Girard, A. Dereux, D. Andre, A. Castiaux and J.-P. Vigneron, Phys. Low-Dim. Struct. 4/5 (1995) 53. 